\title{
Isolation and characterization of multidrug-resistant side population cells in prostate carcinoma
}

\author{
Yu-qiu Wang, He Tian*, Tong-ju Yang, Li Liu \\ Department of Pharmacy, People's Hospital of Zoucheng City, Shandong Province-273500, PR China
}

*For correspondence: Email: tianheth32@hotmail.com; Tel/Fax: 0086-0537-5250813

Received: 24 November 2015

Revised accepted: 10 October 2016

\begin{abstract}
Purpose: To isolate and characterize cancer stem-like side population (SP) cells from prostate cancer tissues using Hoechst 33342 dye exclusion.

Methods: The presence of SP cells was analyzed in tumor samples by fluorescence activated cell sorting. The cell survival rate and ability for cell self-renewal using the sphere formation assay were evaluated after treatment with multiple drugs.

Results: SP cells in the prostate cancer samples constituted $2.8 \%$, but fell to $0.6 \%$ after treatment with verapamil. The SP cells showed high resistance to drugs such as 5-fluorouracil, cisplatin, paclitaxel (2 $\mu \mathrm{mol} / L)$ and oxaliplatin. The survival rate of SP cells after treatment with these drugs was significantly higher $(p<0.01)$ than that of non-SP cells. Furthermore, the number of spheres generated in serumfree medium was significantly higher in prostate cancer SP cells than in non-SP cells.

Conclusion: The presence of SP cells is responsible for prostate treatment failure and tumor recurrence. Therefore, isolation and characterization of SP cells may provide new insights into the development of novel therapeutic agents targeting cancer stem cells for complete eradication of the tumor.
\end{abstract}

Keywords: Side population cells, ABC transporters, Cancer stem cells, Chemotherapy, Prostate treatment failure, Tumor recurrence, Drug resistance

\begin{abstract}
Tropical Journal of Pharmaceutical Research is indexed by Science Citation Index (SciSearch), Scopus, International Pharmaceutical Abstract, Chemical Abstracts, Embase, Index Copernicus, EBSCO, African Index Medicus, JournalSeek, Journal Citation Reports/Science Edition, Directory of Open Access Journals (DOAJ), African Journal Online, Bioline International, Open-J-Gate and Pharmacy Abstracts
\end{abstract}

\section{INTRODUCTION}

Prostate cancer is the most common cancer among males and one of the leading causes of death worldwide [1]. Treatment includes androgen deprivation therapy and chemotherapy based on the cancer stage after diagnosis. However, prostate cancer cells are highly drugresistant, which ultimately results in treatment failure and a high mortality rate. The major implications of drug resistance in tumor biology are often associated with chemotherapy failure, recurrence of the tumor and metastasis.
Cancer stem cells (CSCs) have been identified as a small subset of cells within a tumor population. Several studies have demonstrated that the presence of CSCs is responsible for chemotherapy resistance and treatment failure. The biological characteristics of CSCs, such as self-renewal and a high proliferation rate, are similar to those of normal stem cells, which could have a detrimental effect on cancer treatment [24]. Therefore, understanding the mechanism of CSCs in tumor relapse, as well as improved cancer treatments that specifically target and kill CSCs, are urgently needed in the current field of cancer treatment. 
However, the isolation of such small CSC populations is challenging due to the lack of specific surface markers. The isolation and characterization of side population (SP) cells by Hoechst 33342 dye exclusion is useful in the characterization and targeting of CSCs. These $\mathrm{SP}$ cells share biological characteristics with CSCs, such as self-renewal, increased cell proliferation, multidrug resistance (MDR) and reduced apoptosis. In 1996, Goodell et al. [5] isolated hematopoietic stem cells from bone marrow by the Hoechst 33342 dye exclusion method. Subsequently, SP cells were identified in, and isolated from, several cancer types by Hoechst 33342 dye exclusion using fluorescence activated cell sorting (FACS) [6].

Similar to several other cancers, the presence of CSCs in prostate cancer may be the root cause of treatment failure, cancer metastasis and tumor recurrence. Prostate CSCs undergo abnormal differentiation and form heterogeneous tumor cells with high tumorigenicity and unregulated cell proliferation. In addition, CSCs express stem cell surface markers, such as CD44, CD133, CD147 and ALDH1, and elevated expression of adenosine triphosphate binding cassette (ABC) transporters [7] and anti-apoptotic factors, which contribute to self-renewal, MDR and reduced apoptosis $[8,9]$. Therefore, characterization and understanding of the biological functions of prostate CSCs might provide further insight into the mechanism of drug resistance and help identify useful therapeutic targets to improve the current therapeutic modalities.

Consequently, the aims of the present work were to isolate CSCs from prostate cancer based on Hoechst 33342 dye exclusion and to identify novel therapeutic agents targeting CSCs for complete eradication of the tumor.

\section{EXPERIMENTAL}

\section{Sample collection and cell culture}

Primary prostate cancer tissues were collected from 10 patients with lymph node metastases at the time of surgery (radical prostatectomy) following ethical approval from Southern Medical University, Guangzhou, Guangdong, China. The cancer tissues were washed extensively in PBS containing antibiotics and incubated overnight in DMEM/F12 (Gibco) containing penicillin (500 $\mathrm{U} / \mathrm{mL})$, streptomycin $(500 \mu \mathrm{gg} / \mathrm{mL})$ and amphotericin B $(1.25 \mu \mathrm{g} / \mathrm{mL})$ (Gibco) at $37^{\circ} \mathrm{C}$. Enzymatic digestion was performed using collagenase $(1.5 \mathrm{mg} / \mathrm{mL}) \quad$ (Gibco) and hyaluronidase $(20 \mu \mathrm{g} / \mathrm{mL})$ in PBS for $1 \mathrm{~h}$. Cells were cultured in DMEM supplemented with $10 \%$ FBS and antibiotics and maintained in T75 flasks at $37{ }^{\circ} \mathrm{C}$ in a humidified $5 \% \mathrm{CO}_{2}$ and $95 \%$ air atmosphere. Upon reaching $90 \%$ confluency, the cells were removed from the culture flask using trypsin (0.25\%)-EDTA (53 mM), washed and suspended in $10 \%$ DMEM. The cells were counted using a hemocytometer. The study was approved by Medical Ethics Committee of the People's Hospital of Zoucheng (approval ref no. ZC2010091) and followed the guidelines of Council for International Organizations of Medical Sciences (CIOMS) [10].

\section{Labeling with Hoechst 33342 dye}

The study groups included control cells labeled with Hoechst 33342 dye alone $(n=3)$ and treatment cells labeled with Hoechst 33342 dye and treated with verapamil $(n=5)$. The Hoechst 33342 dye labeling protocol was obtained from Dr. Wanshan Li, Department of Oral and Maxillofacial Surgery, Chongqing Medical University by personal communication.

Cells were counted using a hemocytometer, and approximately $10^{6}$ cells $/ \mathrm{mL}$ in $10 \%$ DMEM were labeled with bisbenzimide Hoechst 33342 stock solution (5 $\mu \mathrm{L} / \mathrm{mL}$; Sigma), either alone or in combination with verapamil $(0.8 \mu \mathrm{L} / \mathrm{mL})$. Cells were then counterstained with $2 \mu \mathrm{gg} / \mathrm{mL}$ propidium iodide. The cells were sorted using a flow cytometer, and the sorted cells were cultured and maintained in DMEM/F-12 supplemented with $10 \%$ FBS. The Hoechst 33342 dye was excited at $355 \mathrm{~nm}$, and its dual wavelength fluorescence was analyzed (blue, $450 \mathrm{~nm}$; red, $675 \mathrm{~nm}$ ).

\section{Immunofluorescence staining}

The immunostaining of spheroids was performed as described previously [11]. Spheroids were fixed onto glass slides using ice-cold $4 \%$ paraformaldehyde $\left(4{ }^{\circ} \mathrm{C}, 10 \mathrm{~min}\right)$, blocked with normal serum for 30 min and incubated with mouse monoclonal anti-Oct4 (1:200; Chemicon, Japan) overnight. After washing the slides with PBS, they were incubated with FITC-conjugated chicken anti-rat IgG overnight in a dark room. Nuclei were counterstained with Hoechst 33342 dye and viewed by fluorescence microscopy, and the resulting images were processed using Image $\mathrm{J}$ software (ver. 1.4; $\mathrm{NIH}$ ).

\section{Drug resistance assay}

The cells were assayed for drug resistance as described previously [5]. Approximately $1 \times 10^{3}$ 
cells/plate were cultured in 96-well plates and treated with 5-fluorouracil (5-FU) (10 $\mu \mathrm{g} / \mathrm{mL})$, cisplatin $(20 \mu \mathrm{mol} / \mathrm{L})$, paclitaxel $(2 \mu \mathrm{mol} / \mathrm{L})$ and oxaliplatin (100 mM). The absorbance at $450 \mathrm{~nm}$ was obtained spectrophotometrically. Cell resistance to the drugs was calculated for both groups using Eq 1 [12].

Cell resistance $(\%)=(\mathrm{Ae} / \mathrm{Ac}) 100$

where $\mathrm{Ae}$ and $\mathrm{Ac}$ are the absorbances of the experimental and control groups, respectively.

\section{Sphere formation assay}

The sphere formation assay was performed exactly as described previously. The sorted SP and non-SP cells were resuspended at a density of $1,000 / \mathrm{mL}$ in serum-free tumor sphere medium consisting of Ham's F-12/DMEM (1:1), $10 \mathrm{ng} / \mathrm{mL}$ human recombinant bFGF and $10 \mathrm{ng} / \mathrm{mL}$ EGF and were subsequently cultured in ultra-low attachment plates for approximately 2 weeks. The sorted SP and non-SP cells were seeded at a low density of $20 / L$, and the number of spheres generated was counted after 7 days of culture.

\section{Statistical analysis}

One-way analysis of variance (ANOVA) and Student's t-test were performed to determine significant differences between the treatment and control groups. $P<0.05$ was considered statistically significant. Statistical analysis was performed using GraphPad Prism software (ver. 6.0; GraphPad Software).

\section{RESULTS}

\section{Side population cells}

As shown in the dot plot generated from the FACS data (Figure 1), the proportion of SP cells observed was approximately $2.8 \%$. The number of cells identified was approximately 59,000, which was approximately $55 \%$ of the initial cell count. Of the 59,000 cells (P1) analyzed, $2.8 \%$ showed Hoechst dye efflux in the P2 gated region of the dot plot (Figure 1) Treatment with verapamil, an inhibitor of the MDR1 transporter, resulted in a reduced proportion $(0.6 \%)$ of SP cells (Figure 2).
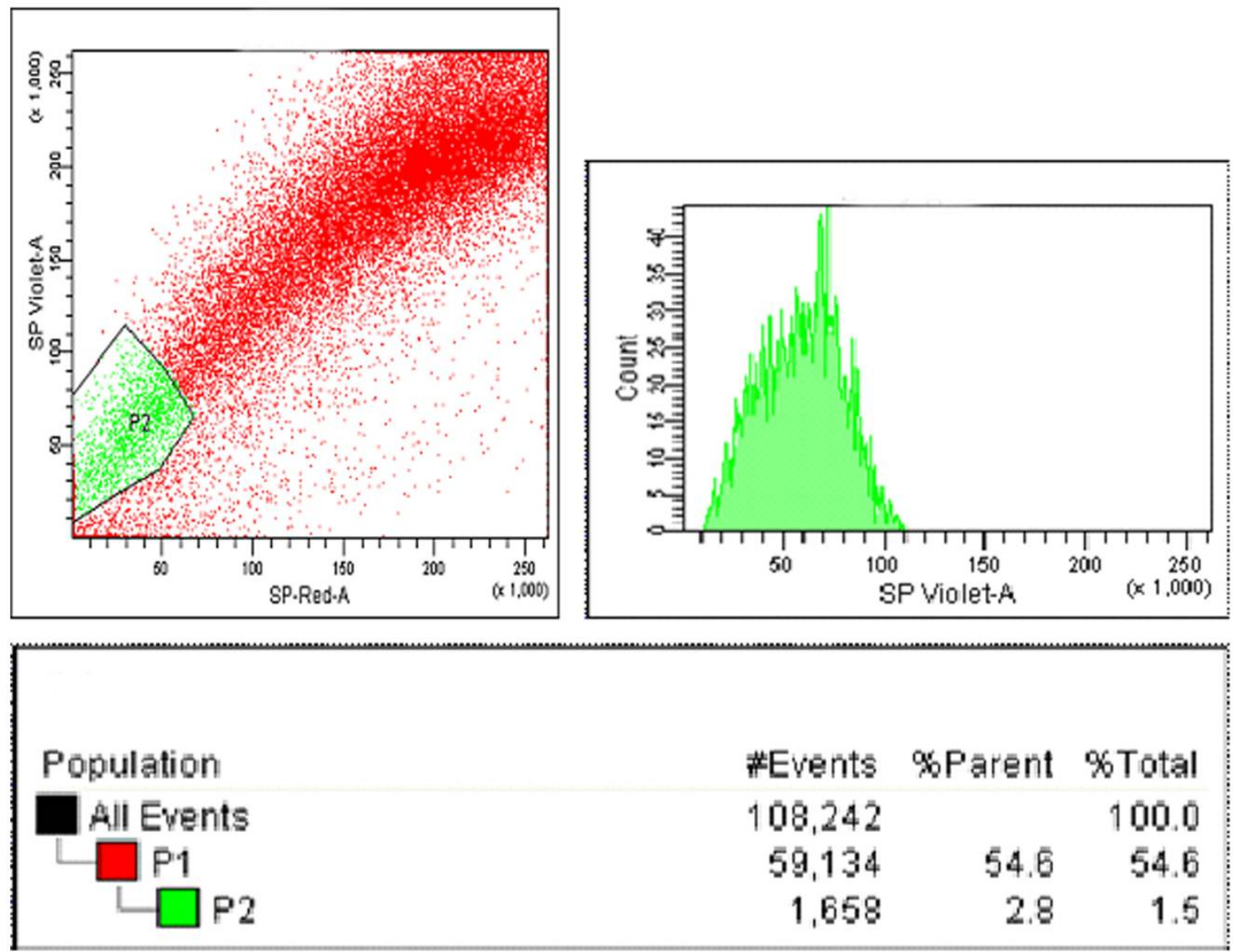

Figure 1: Representative dot plot analysis by fluorescence activated cell sorting (FACS) showing the presence of $2.8 \%$ side population (SP) cells in prostate cancer samples in the P2 gated region 

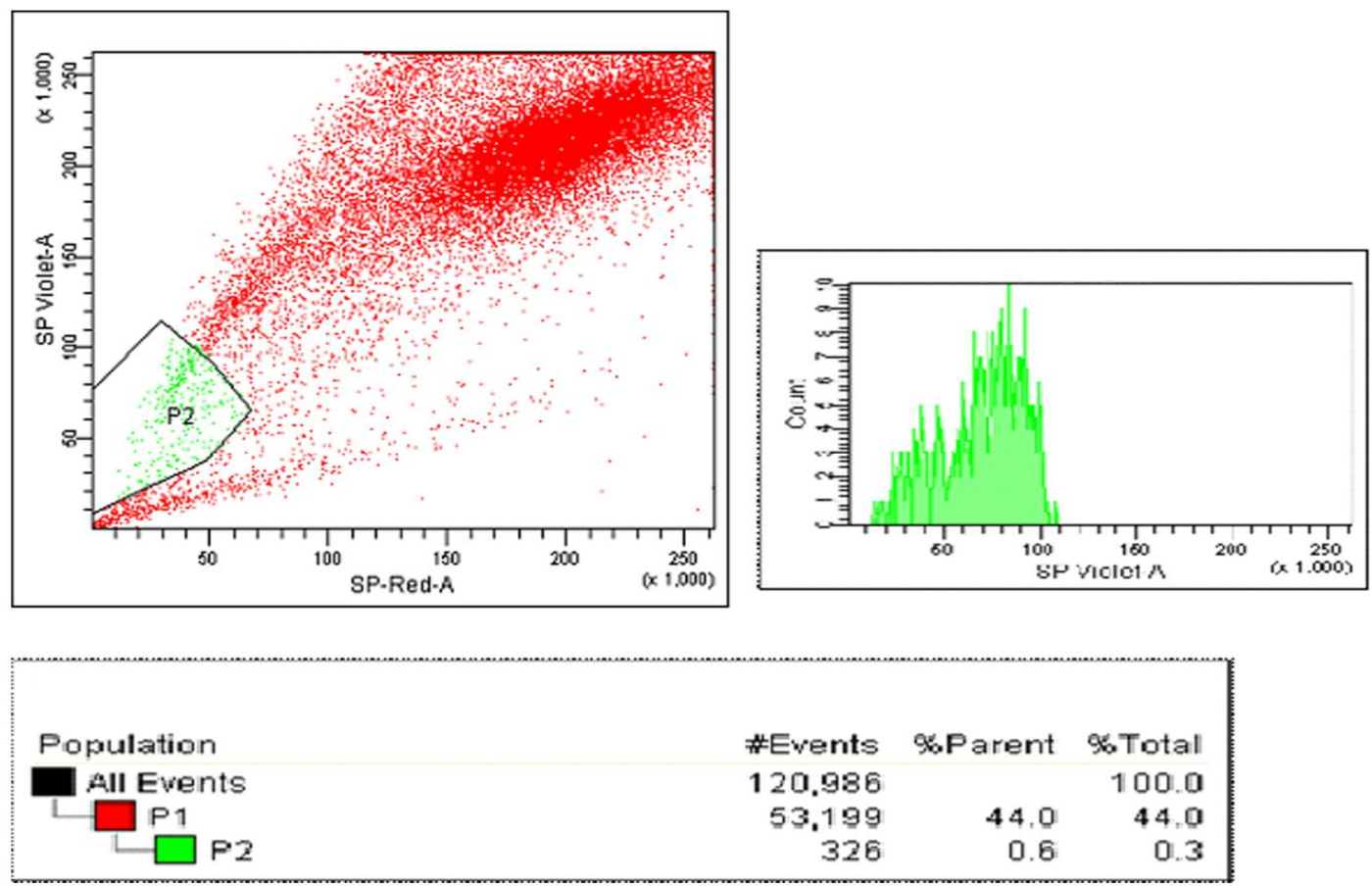

Figure 2: Representative dot plot analysis by FACS showing the reduction of SP cells $(0.6 \%)$ after treatment with verapamil. Note: The proportion of SP cells was reduced in the P2 gated region

A

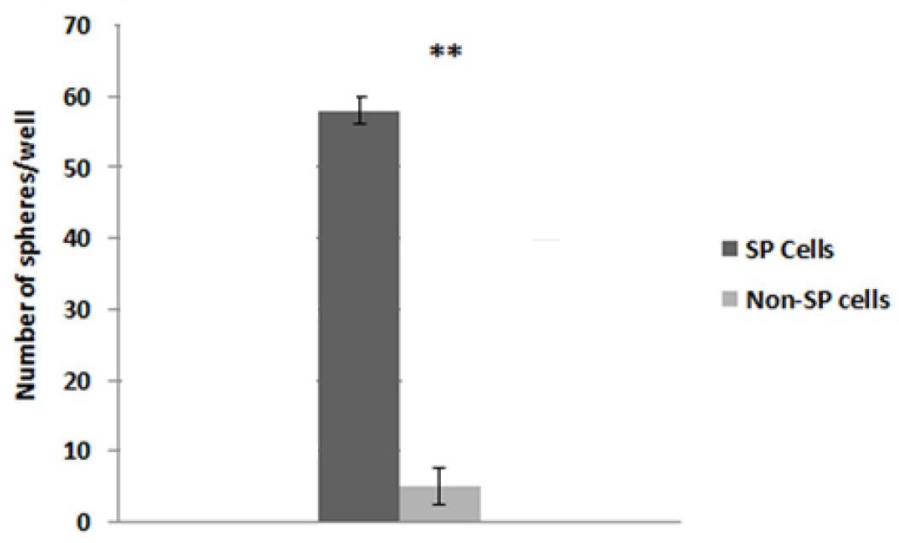

B

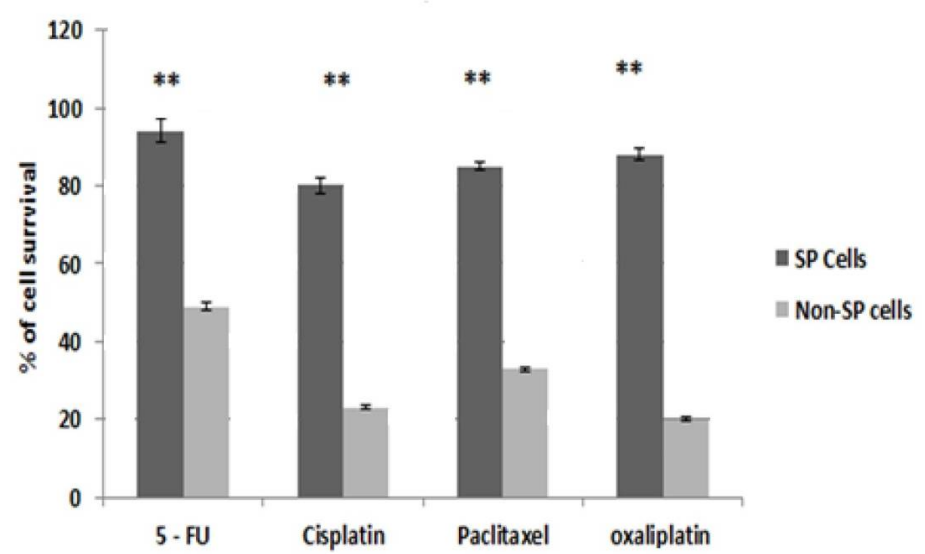

Figure 3: (A) Quantification of the number of spheres generated by the sorted SP and non-SP cells, which were counted after 7 days of culturing. The SP cells produced significantly more tumor spheres than did non-SP cells. (B) Comparison of cell survival rates between SP and non-SP cells after treatment with 5-fluorouracil, cisplatin, paclitaxel and oxaliplatin 

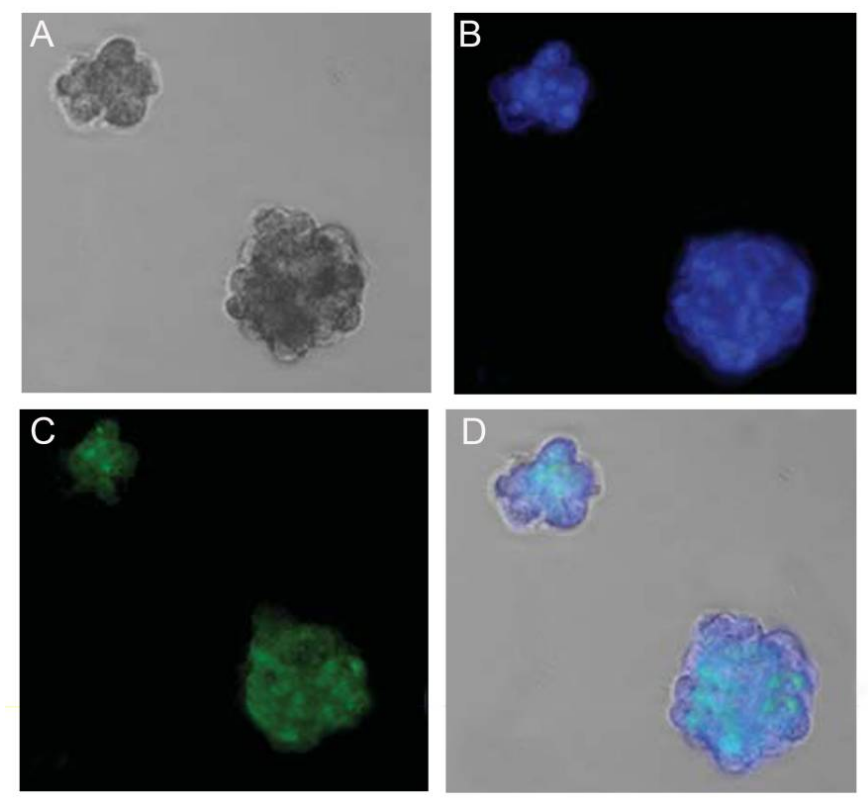

Figure 4: Representative immunostaining of Oct4 in tumor spheres generated by prostate cancer SP cells. (A) Bright field micrographs of the sphere cells. (B) Fluorescent Hoechst 33342 staining (blue) of the nucleus. (C) Immunostaining of anti-Oct4 (green fluorescence). (D) Overlay of the images from (B) and (C)

\section{Characteristics of SP cells derived from prostate cancer}

After carefully analyzing the presence of SP cells by FACS, the SP and non-SP cells were sorted separately by FACS. To examine the chemoresistant phenotype and self-renewing capacity of the CSCs, the sorted SP and non-SP cells were subjected to drug resistance and sphere formation assays. The SP cells showed high resistance to drugs such as 5-FU, cisplatin, paclitaxel $(2 \mu \mathrm{mol} / \mathrm{L})$ and oxaliplatin. The survival rate of SP cells after treatment with these drugs was significantly higher $(p<0.01)$ than that of non-SP cells (Figure $3 \mathrm{~A}$ ). Furthermore, the number of spheres generated in serum-free medium was significantly higher in prostate cancer SP cells than in non-SP cells (Figure 3B). We also found that the spheres formed by SP cells were Oct4-positive by immunofluorescence (Figure 4A-D). From these data, it is clear that prostate cancer SP cells exhibit high MDR and proliferation potential.

\section{DISCUSSION}

CSCs identified in several cancers appear to resist DNA-damaging agents due to high expression of efflux pumps and enhanced DNA repair capacity. These properties may explain the long-term treatment failure of standard chemotherapies, which initially result in tumor shrinkage by killing most neoplastic cells; however, the cancer eventually relapses due to the robust nature and resistance of CSCs [1-5]. Most traditional treatments have the ability to kill the majority of the tumor population, with the exception of CSCs, which are highly resistant to chemotherapy. These resistant CSCs are responsible for tumor growth, metastasis and relapse. Hence, the elimination of CSCs is an important goal in eradicating refractory cancers and providing long-term disease-free survival.

One of the characteristic features of CSCs used for their identification is their capacity to extrude dyes such as Hoechst 33342. Cells that exclude this dye are referred to as SP cells [6]. These cells share characteristics with CSCs; specifically, both cell types exhibit a capacity for tumor initiation, expression of stem-like genes, and resistance to chemotherapeutic drugs. Dye exclusion is a valuable technique, as it identifies a unique population of cells with stem-like characteristics.

According to recent findings, SP cells are present in various types of cancer, including cancers of the blood, liver, lung, skin, nasopharyngeal, ovary and central nervous system [13-17]. SP cells share fundamental and similar biological characteristics with CSCs, such as differentiation potential, deregulation of the cell cycle, high tumorigenic ability and resistance to chemotherapeutic drugs. However, the use of various markers for isolating SP cells has been strongly debated. In the current study, we used 
the Hoechst 33342 dye exclusion technique for identification and isolation of SP cells from prostate cancer. Similar to other tumors, SP cells are present in prostate tumors.

We identified SP cells in our prostate cancer samples at a prevalence of $2.8 \%$, which was reduced to $0.6 \%$ after treatment with verapamil. Therefore, Hoechst 33342 dye exclusion can be used to isolate SP cells. The exclusion of Hoechst 33342 dye from SP cells is most likely the result of overexpression of MDR transporters, such as $A B C B 1$ and $A B C G 2$, which might be responsible for drug resistance and treatment failure. The sorted SP cells were highly resistant to various drugs including 5-FU, cisplatin, oxaliplatin and paclitaxel, and the survival rate of SP cells after treatment with these drugs was significantly higher compared with that of non-SP cells. This increased survival rate could be explained by two different mechanisms: i) activation and overexpression of $A B C$ transporters (e.g., ABCB1 and ABCG2), which expel harmful mate rials from cells, and ii) elevated expression of anti-apoptotic factor bcl-2, which ultimately results in reduced apoptosis [18]. Similar to previous findings in gastric cancer cell lines, we speculate that both processes occur in SP cells.

The most important feature of CSCs is their strong ability to form tumor spheres, which can also be used as a marker to isolate CSCs. We clearly showed that isolated SP cells form tumor spheres positive for Oct4 immunostaining. Oct4 expression suggests that SP cells have a high capacity for self-renewal. These SP cells are hypothesized to exhibit increased expression of stem cell surface markers, such as CD44/CD147 and bcl-2, which confer significant prostate cancer metastasis, self-renewal and apoptotic resistance capabilities, respectively. These research questions have yet to be answered. Therefore, characterization of SP cells enables the design of novel therapeutic agents that selectively target CSCs and improve cancer therapy by blocking $A B C$ transporters. Furthermore, the differences in signaling pathways between normal cells and CSCs need to be elucidated before novel therapeutic targets with the eventual goal of eliminating residual disease and recurrence are achievable.

\section{CONCLUSION}

The presence of SP cells is responsible for prostate cancer treatment failure and tumor recurrence. Therefore, isolation and characterization of SP cells may provide new insights into the development of novel therapeutic agents targeting CSCs for complete eradication of tumors.

\section{DECLARATIONS}

\section{Acknowledgement}

The authors thank Dr. Wanshan Li, Department of Oral and Maxillofacial Surgery, Chongqing Medical University for sharing FACS protocol by personal communication.

\section{Conflict of Interest}

No conflict of interest associated with this work.

\section{Contribution of Authors}

The authors declare that this work was done by the authors named in this article and all liabilities pertaining to claims relating to the content of this article will be borne by them.

\section{Open Access}

This is an Open Access article distributed under the terms of the Creative Commons Attribution License, which permits unrestricted use, distribution, and reproduction in any medium, provided the original work is properly credited.

\section{REFERENCES}

1. Jemal A, Siegel R, Ward E, Hao Y, Xu J, Murray T, Thun MJ. Cancer statistics. CA Cancer J Clin 2008; 58: 71-96.

2. Yang JP, LiU $Y$, Zhong $W$, Yu $D$, Wen $L J$, Jin $C S$. Chemoresistance of CD 133+ cancer stem cells in laryngeal carcinoma. Chin Med J (Engl) 2011; 124: 1055-1060.

3. Scaldaferri ML, Fera S, Grisanti L, Sanchez M, Stefanini $M$, De Felici $M$, Vicini E. Identification of side population cells in mouse primordial germ cells and prenatal testis. Int J Dev Biol 2011; 55: 209-214.

4. Takao T, Asanoma K, Kato K, Fukushima K, Tsunematsu $R$, Hirakawa T, Matsumura S, Seki H, Takeda S, Wake $N$. Isolation and characterization of human trophoblast side population (SP) cells in primary villous cytotrophoblasts and HTR-8/SVneo cell line. PLoS One 2011; 6: e21990.

5. Goodell MA, Brose K, Paradis G, Conner AS, Mulligan $R C$. Isolation and functional properties of murine hematopoietic stem cells that are replicating in vivo. $J$ Exp Med 1996; 183: 1797-1806.

6. Hirschmann-Jax C, Foster AE, Wulf GG, Nuchtern JG, Jax TW, Gobel U, Goodell MA, Brenner MK. A distinct side population of cells with high drug efflux 
capacity in human tumor cells. Proc Natl Acad USA 2004; 101: 14228-14233.

7. Bourguignon LY, Peyrollier K, Xia W, Gilad E. Hyaluronan-CD44 interaction activates stem cell marker Nanog, Stat-3-mediated MDR1 gene expression, and ankyrin-regulated multidrug efflux in breast and ovarian tumor cells. J Biol Chem 2008; 283: 17635-17651.

8. Gao AC, Lou W, Sleeman JP, Isaacs JT. Metastasis suppression by the standard CD44 isoform does not require the binding of prostate cancer cells to hyaluronate. Cancer Res 1998; 58: 2350-2352.

9. Yan L, Zucker S, Toole BP. Roles of the multifunctional glycoprotein, emmprin (basigin; $C D$ 147), in tumour progression. Thromb Haemost 2005; 93: 199-204.

10. Council for International Organizations of Medical Sciences, World Health Organization. International Ethical Guidelines for Biomedical Research Involving Human Subjects. Geneva, Switzerland: World Health Organization; 2002.

11. Ma L, Lai D, Liu T, Cheng W, Guo L. Cancer stem-like cells can be isolated with drug selection in human ovarian cancer cell line SKOV3. Acta Biochim Biophys Sin (Shanghai) 2010; 42: 593-602.

12. He QZ, Luo XZ, Wang K, Zhou Q, Ao H, Yang Y, Li SX, Li Y, Zhu HT, Duan T. Isolation and characterization of cancer stem cells from high-grade serous ovarian carcinomas. Cell Physiol Biochem 2014; 33: 173-184.
13. Yanamoto S, Kawasaki G, Yamada S, Yoshitomi I, Kawano $T$, Yonezawa $H$, Rokutanda $S$, Naruse $T$, Umeda M. Isolation and characterization of cancer stem-like side population cells in human oral cancer cells. Oral Oncol 2011; 47: 855-860.

14. Shimano K, Satake M, Okaya A, Kitanaka J, Kitanaka N, Takemura M, Sakagami M, Terada N, Tsujimura $T$. Hepatic oval cells have the side population phenotype defined by expression of ATP binding cassette transporter ABCG2/BCRP1. Am J Pathol 2003; 163: 39.

15. Bapat SA, Mali AM, Koppikar CB, Kurrey NK: Stem and progenitor-like cells contribute to the aggressive behavior of human epithelial ovarian cancer. Cancer Res 2005; 65: 3025-3029.

16. Wang J, Guo LP, Chen LZ, Zeng YX, Lu SH. Identification of cancer stem cell-like side population cells in human nasopharyngeal carcinoma cell line. Cancer Res 2007; 67: 3716-3724.

17. Singh SK, Hawkins C, Clarke ID, Squire JA, Bayani J, Hide T, Henkelman RM, Cusimano MD, Dirks PB. Identification of human brain tumour initiating cells. Nature 2004; 432: 396-401.

18. Li Y, Rizvi SM, Ranson M, Allen BJ. 213Bi-PAl2 conjugate selectively induces apoptosis in PC3 metastatic prostate cancer cell line and shows anticancer activity in a xenograft animal model. Br J Cancer 2002; 86: 1197-1203. 